\title{
Le programme Survivors Teaching Students : une sensibilisation accrue au cancer de l'ovaire
}

par Margaret Fitch, inf., Ph.D., Alison McAndrew, B.A., RAP, Fran Turner, M.S., Elisabeth Ross, M.H.Sc., et Iris Pison, B.Sc., MBA

\section{Abrégé}

De nouvelles données probantes révèlent la présence persistante d'une combinaison de symptômes pouvant indiquer l'existence d'un cancer de l'ovaire. Les professionnels de la santé doivent être au courant de ces données probantes et les incorporer dans leur pratique. L'organisme Cancer de l'ovaire Canada (COC) a mis en oeuvre un programme novateur dans diverses universités canadiennes en tant que méthode visant à instruire les étudiants de premier cycle en médecine et en sciences infirmières sur le cancer de l'ovaire. Le programme Survivors Teaching Students a été dispensé à 3620 étudiants de premier cycle. Le présent article présente l'évaluation du programme réalisée au moyen de sondages menés avant et après les séances. Dans l'ensemble, les étudiants ont fait état d'une amélioration de leurs connaissances sur l'évolution du cancer de l'ovaire, les symptômes de ce type de cancer, les facteurs de risques afférents et aussi de leurs perspectives sur ce qu'une femme atteinte d'un cancer de l'ovaire pouvait bien ressentir à l'annonce du diagnostic. En demandant à des survivantes de présenter l'exposé en classe, les étudiants ont pu mettre "un visage et une voix » sur l'enjeu qu'est le cancer de l'ovaire.

\section{Au sujet des auteures}

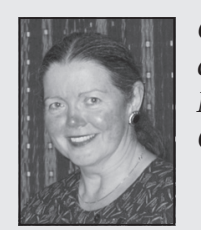

Coordonnées : Margaret Fitch, inf., Ph.D., Centre de cancérologie Odette, 2075 Bayview Ave., Toronto, ON M4N 3M5. Tél. : 416-480-5891; Téléc. : 416-480-7806; Courriel : marg.fitch@sunnybrook.ca

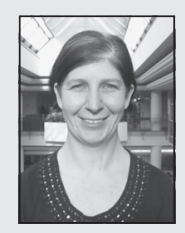

Alison McAndrew, B.A., RAP, Centre de cancérologie Odette, 2075 Bayview Ave., Toronto, ON M4N 3M5. Courriel : alison.mcandrew@sunnybrook.ca

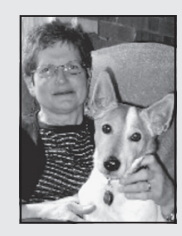

Fran Turner, M.S., Ovarian Cancer Canada, 145 Front Street East, Suite 101, Toronto, ON M5A $1 E 3$. Courriel : fturner@ovariancanada.org

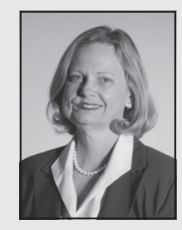

Elisabeth Ross, M.H.Sc., Ovarian Cancer Canada, 145 Front Street East, Suite 101, Toronto, ON M5A 1E3. Courriel : eross@ovariancanada.org

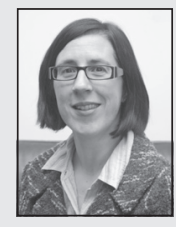

Iris Pison, B.Sc., MBA, Ovarian Cancer Canada, 145 Front Street East, Suite 101, Toronto, ON M5A $1 E 3$. Courriel : ipison@ovariancanada.org

\section{Introduction}

Les femmes du Canada ont, à vie, une chance sur 70 d'être diagnostiquées d'un cancer de l'ovaire (INCC, 2009). Environ 2700 diagnostics de ce cancer sont posés chaque année. Le cancer de l'ovaire est le plus grave des cancers gynécologiques; à la $5^{\mathrm{e}}$ place des cancers chez les femmes par sa fréquence, il se classe au $4^{\mathrm{e}}$ rang des causes de mortalité. On l'a surnommé «maladie silencieuse » parce que ses signes et ses symptômes sont plutôt vagues et subtils, ce qui se traduit par des diagnostics de cancer au stade avancé dont le taux de survie ne s'élève qu'à $20 \%$ (Jayde, White \& Blomfield, 2009). Toutefois, de récentes études ont permis de cerner la présence persistante d'une combinaison de symptômes (p. ex. ballonnement, augmentation du volume de l'abdomen et symptômes urinaires) pouvant indiquer l'existence d'un cancer de l'ovaire (Bankhead et al., 2008; Devlin et al., 2010; Goff, Mandel, Melancon \& Muntz, 2004; Hamilton, Peters, Bankhead \& Sharp, 2009). En l'absence d'un test de dépistage fiable (Andersen et al., 2008; Jayde et al., 2009; Rossing, Wicklund, Cushing-Haugen \& Weiss, 2010), la présence de cette combinaison de symptômes justifie la réalisation d'examens cliniques en vue de voir s'il s'agit d'un cancer de l'ovaire. Les professionnels de la santé doivent non seulement être au courant de ces nouveaux résultats mais encore ils doivent les incorporer dans leur pratique. Des initiatives pédagogiques sont nécessaires en vue d'atteindre ce but aussi bien au niveau du premier cycle qu'à celui des cycles supérieurs.

\section{Contexte}

La nécessité de sensibiliser davantage les professionnels de la santé aux signes et symptômes du cancer de l'ovaire et aux facteurs de risque d'origine familial pouvant indiquer une prédisposition au cancer de l'ovaire ou au cancer du sein, a été dégagée dans deux enquêtes nationales effectuées l'une auprès des médecins de famille (Gray, Chart, Carrol, Fitch \& Cloutier-Fisher, 1999) et l'autre auprès de survivantes du cancer ovarien (Fitch, Gray, DePetrillo, Franssen \& Howell, 1999). En particulier, les survivantes décrivaient les défis qu'elles devaient surmonter afin d'obtenir une aide professionnelle relativement aux symptômes qu'elles éprouvaient et afin d'obtenir un diagnostic formel (Fitch, Deane, Howell \& Gray, 2002). Ces résultats ont été présentés lors de la première conférence pancanadienne sur le cancer de l'ovaire, Forum sur le cancer de l'ovaire 1999 et ont stimulé les initiatives pédagogiques visant le grand public et les professionnels de la santé. Les survivantes, les chercheurs, les professionnels de la santé et les autres intervenants présents au forum ont préparé un mandat à trois volets pour Cancer de l'ovaire Canada : 1) appuyer les femmes atteintes de la maladie et leurs familles, 2) sensibiliser le grand public et les professionnels de la santé, 3) financer la recherche pour mettre au point des techniques de dépistage précoce et améliorer le traitement. Depuis ce temps-là, ces orientations guident le personnel de l'organisation dans la planification des programmes et de l'extension des services à la communauté (voir http://ovariancanada.org/?lang=fr-CA).

Les premiers efforts de sensibilisation des professionnels de la santé visaient avant tout les médecins de famille. Cancer de l'ovaire Canada a élaboré un cours en ligne en partenariat avec l'Université McMaster (Ontario) et l'Université Memorial (Terre-Neuve). Il s'agit du cours fourni à http://www.mdcme.ca/cmecourse_info. asp?Id=42 qui est offert de manière continue et gratuite aux médecins de famille. À l'heure actuelle, COC met au point, à titre expérimental et à l'intention des médecins de famille, un cours donné en face à face qui fait appel à des survivantes. 
L'initiative de sensibilisation suivante a étendu les efforts de Cancer de l'ovaire Canada aux étudiants de disciplines de la santé. L'initiative a pris la forme d'un nouveau programme canadien baptisé Survivors Teaching Students [Les survivantes enseignent aux étudiants]. Cet article a pour but de décrire le programme et de présenter les résultats de son évaluation après ses deux premières années de mise en œuvre dans des universités canadiennes auprès d'étudiants de premier cycle en médecine et en sciences infirmières. L'on s'attend à ce que ce type de programme soit utile dans d'autres disciplines et pour d'autres sièges du cancer.

\section{Survivors Teaching Students: description du programme}

\section{Le programme original}

Le programme baptisé Survivors Teaching Students (STS) Saving Women's Lives avait été institué en 2002 aux États-Unis par Betty Reiser de l'University of Medicine and Dentistry of New Jersey. Il a fini par devenir le programme vedette de l'Ovarian Cancer National Alliance, un organisme américain qui défend les intérêts des femmes atteintes d'un cancer ovarien (www.ovariancancer.org). Dorénavant, le programme est offert dans 60 écoles de médecine et universités de l'ensemble des États-Unis.

Le programme STS a été conçu afin de former les femmes diagnostiquées d'un cancer de l'ovaire à donner des présentations sur leurs expériences de la maladie à des étudiants œuvrant dans des disciplines de la santé. Les survivantes donnent le programme en groupes de trois. Les idées clés présentées aux étudiants sont que la majorité des cas de cancer ovarien ne sont pas diagnostiqués avant que la maladie ne soit parvenue aux stades avancés où la survie est compromise. Ceci s'explique, en grande partie, par le fait qu'il est possible que les symptômes qui se présentent ne soient pas reconnus comme étant des symptômes annonciateurs du cancer de l'ovaire. En outre, il se peut que les femmes courant un risque accru ne soient pas identifiées ni surveillées comme il faudrait (Ryerson et al., 2007; Bankhead et al., 2008). Ces idées sont intégrées par les survivantes dans leurs récits sur leurs expériences en matière de cancer de l'ovaire. STS fait entrer le visage et la voix des survivantes du cancer de l'ovaire dans les salles de cours des étudiants de premier cycle en médecine, sciences infirmières, pharmacie ou travail social. Les présentations confèrent, dans la salle de cours, une bonne dose de réalité au cancer ou un exemple bien vivant du vécu du cancer. Les présentations sont conçues pour faciliter l'apprentissage, tôt dans la carrière professionnelle des étudiants des disciplines de la santé, des points de vue ou des perspectives des patients et des survivants. Elles ont également été élaborées pour sensibiliser les étudiants à la manière d'annoncer une mauvaise nouvelle et à aborder valablement les statistiques lors de la discussion du pronostic.

\section{Importation du programme au Canada}

Cancer de l'ovaire Canada a importé le programme STS en 2006. Son contenu a été canadianisé comme il le fallait, et des survivantes ont été recrutées et formées en vue de devenir des présentatrices du programme. Betty Reiser a assuré elle-même la formation des 70 premières survivantes canadiennes et COC a ensuite pris le relais. L'atelier de formation utilisé comprenait deux éléments primaires. Le premier était d'apprendre à présenter et à renforcer les messages primordiaux sur le cancer de l'ovaire (voir ci-dessous). Le second était de peaufiner le récit écrit que la survivante préparait sur son expérience en matière de symptômes, sur la façon dont elle avait été diagnostiquée, sur ses antécédents familiaux et ses progrès au fil du traitement. Ces récits incorporent les sentiments et les perspectives des femmes relativement à leurs interactions avec les professionnels de la santé. Pendant la période de formation, les survivantes se sont entraînées à donner leurs présentations et à répondre aux questions de leur auditoire. La séance de formation durait habituellement 50 minutes.

\section{Sélection des survivantes qui recevront la formation}

C'est dans les publications de COC (Semons l'espoir et son bulletin électronique) qu'un appel a été lancé aux survivantes intéressées à participer bénévolement au programme STS. L'invitation précisait les endroits particuliers puisque la formation des survivantes allait avoir lieu dans des villes possédant des écoles de médecine et de sciences infirmières. Il était préférable que les survivantes faisant les présentations aux étudiants reflètent une gamme étendue de stades au moment du diagnostic et diverses durées de survivance. Quoiqu'il fût souhaitable de recruter des femmes qui ne sont pas aux prises avec un cancer actif, la réalité veut que beaucoup des survivantes qui se portent volontaires sont susceptibles d'éprouver des récidives, et certaines d'entre elles sont d'ailleurs décédées au cours du programme. Les femmes qui désiraient participer au programme étaient priées de contacter la directrice des programmes de COC et de rédiger et d'envoyer le récit de leur expérience du cancer et ce, avant qu'elles ne soient retenues pour une entrevue. Cette dernière incluait l'examen du récit de la femme et une discussion des lignes directrices du programme. Il importe que les candidates comprennent bien ce qui sera exigé d'elles si on leur demande de participer à Survivors Teaching Students.

Les survivantes bénévoles ont exprimé une rétroaction très positive sur l'atelier de formation de STS et sur la manière de présenter leurs récits (McAndrew, Fitch, Turner \& Ross, 2008). Dans l'ensemble, elles voyaient dans le programme une occasion d'influencer les professionnels de la santé de demain relativement au cancer de l'ovaire et à ses symptômes. En particulier, la formation les aidait à se sentir à l'aise pour présenter leur histoire personnelle à un auditoire composé d'étudiants. [Voir l'encadré A sur les perspectives des bénévoles.]

Encadré A. Perspectives des survivantes bénévoles au sujet de STS

La motivation des survivantes à participer au programme Je voulais avoir l'impression de faire quelque chose qui puisse aider les femmes à l'avenir - en particulier parce que je suis porteuse du gène mutant BRCA2.Je veux essayer d'avoir un impact sur la détection précoce pour le bien de mes enfants déjà adultes.

Étant donnée l'expérience positive que j'ai eue tout au long du l'étape pré-diagnostic, j'estimais qu'il importait d'aider d'autres membres du personnel médical à réaliser à quel point il importe : (a) de savoir identifier rapidement le cancer ovarien, b) d'y répondre de manière positive et bienveillante.

J'ai tant reçu durant ma maladie que je veux donner à mon tour.

La réussite du programme telle que définie par les survivantes Si mon histoire, de concert avec d'autres, a une incidence sur les médecins de demain, de manière à ce qui soient plus à l'écoute des préoccupations des femmes et plus susceptibles de faire figurer le cancer de l'ovaire dans leur liste des maladies à exclure en présence d'une vaste gamme de signes/symptômes.

...sensibiliser [les étudiants en médecine] aux signes subtils du cancer des ovaires et aux besoins/craintes des patients lors du diagnostic et tout au long du traitement.

L'opportunité de partager mon histoire.

Les attentes des survivantes envers ce programme Ouvrir l'esprit des étudiants et les sensibiliser aux histoires de leurs patients.

Faire entrer le cancer ovarien dans une " liste diagnostique différentielle standard » pour les signes abdominaux vagues signalés par les femmes.

Que les étudiants aient entendu notre message. 
Exécution du programme STS

Une fois que le personnel enseignant d'une université accordait sa permission de faire la présentation en salle de cours, chaque séance était donnée selon le format suivant :

- Chaque présentation durait une heure en présence des étudiants.

- Normalement, chaque présentation comprenait trois présentatrices qui étaient des survivantes du cancer de l'ovaire. L'une était chargée de l'animation de la séance. La présentation mettait en jeu, dans la mesure du possible, des femmes ayant des expériences et des antécédents différents. Dans bien des cas, les femmes qui donnaient les présentations avaient été diagnostiquées à un stade avancé, mais il est très utile d'inclure également des femmes dont le diagnostic a été posé à un stade précoce.

- Lors de la séance, l'animatrice présentait une brève introduction et les présentatrices racontaient tour à tour leur histoire, en soulignant la difficulté d'un diagnostic précoce et ce qui leur était arrivé de ce fait. Les récits des survivantes donnaient un visage et une voix à la maladie, un processus qui est censé constituer un puissant outil pour accroître la compréhension des étudiants et fournir à ces derniers l'occasion de découvrir les aspects psychosociaux du cancer de l'ovaire.

- Après chacune des présentations, un dialogue animé par une survivante ouvrait les voies de communication entre les présentatrices et les étudiants et favorisait une interaction directe portant sur les questions de fond.

- Les étudiants ont participé à de brèves enquêtes pré-test et posttest visant à évaluer leur compréhension de la maladie et la valeur de la présentation.

- La présentation était offerte gratuitement aux facultés formant les professionnels de la santé.

\section{Messages clés partagés dans le cadre de la présentation}

Le programme STS vise à véhiculer des messages clés particuliers. Il n'était pas exigé de chaque présentatrice qu'elle aborde chacun d'entre eux mais l'ensemble de la présentation en salle de cours était conceptualisé de manière à souligner tous ces messages. Les

\begin{tabular}{|l|c|c|c|c|}
\hline \multicolumn{1}{|c|}{ Tableau 1. Observations choisies sur l'échantillon } \\
\hline & \multicolumn{2}{|c|}{$\begin{array}{c}\text { Étudiants en } \\
\text { médecine }\end{array}$} & \multicolumn{2}{c|}{$\begin{array}{c}\text { Étudiants en } \\
\text { sciences infirmières }\end{array}$} \\
\cline { 2 - 5 } & Nombre & $\%$ & Nombre & $\%$ \\
\hline $\begin{array}{l}\text { Étudiants ayant } \\
\text { assisté aux } \\
\text { présentations en } \\
\text { salle de cours }\end{array}$ & 798 & 100 & 2822 & 100 \\
\hline $\begin{array}{l}\text { Enquêtes pré- } \\
\text { séance soumises }\end{array}$ & 344 & 43 & 1156 & 41 \\
\hline $\begin{array}{l}\text { Enquêtes post- } \\
\text { séance soumises }\end{array}$ & 352 & 44 & 1203 & 42,6 \\
\hline $\begin{array}{l}\text { Hommes qui ont } \\
\text { soumis l'enquête } \\
\text { pré-séance }\end{array}$ & 121 & $35,2 *$ & 87 & $7,5 *$ \\
\hline $\begin{array}{l}\text { Femmes qui ont } \\
\text { soumis l'enquête } \\
\text { post-séance }\end{array}$ & 215 & $62,5 *$ & 1,027 & $89 *$ \\
\hline $\begin{array}{l}\text { Enquêtes soumises } \\
\text { en ligne }\end{array}$ & 31 & 3,9 & 59 & 2,1 \\
\hline * La proportion est calculée par rapport à l'ensemble des \\
soumissions.
\end{tabular}

messages relatifs aux symptômes sont au cœur même des objectifs du programme. Chaque survivante présentant son histoire dirigeait l'attention sur les aspects de cette dernière qui aident à souligner et à renforcer ces messages clés. Si certains points ne sont pas abordés par les présentatrices de la séance, l'animatrice les soulèvera lors de la discussion tenue avec les étudiants à la suite des présentations individuelles. Les messages clés véhiculés par le programme sont les suivants :

- Le cancer de l'ovaire a le taux de mortalité le plus élevé de tous les cancers gynécologiques et constitue l'une des cinq premières causes de décès par cancer parmi les Canadiennes.

- La majorité des femmes sont diagnostiquées d'un cancer de l'ovaire aux stades avancés.

- Il n'existe présentement aucun test de dépistage fiable pour la détection précoce du cancer de l'ovaire.

- Lorsque ce cancer est détecté aux stades précoces, les taux de survie au cancer de l'ovaire sont nettement meilleurs.

- Les symptômes du cancer de l'ovaire comprennent les suivants :

- ballonnement ou gonflement de l'abdomen;

- douleur ou lourdeur pelvienne;

- douleur au dos ou à l'abdomen;

- fatigue;

- gaz, nausées, indigestion;

- changements du transit intestinal;

- besoin fréquent d'uriner;

- irrégularités du cycle menstruel;

- perte ou gain de poids.

- Si la femme éprouve l'un ou plusieurs de ces symptômes pendant au moins trois semaines, il importe d'entrevoir la possibilité qu'il s'agit du cancer de l'ovaire.

- Si la femme présente des signes et des symptômes indicatifs du cancer de l'ovaire, il convient de réaliser un examen pelvien complet, une échographie transvaginale et une analyse du CA 125 dans le sang.

- Si l'on soupçonne la présence d'un cancer de l'ovaire, les femmes doivent être aiguillées vers un gynéco-oncologue.

- Les femmes ayant des antécédents personnels ou familiaux de cancer des ovaires, du sein ou colorectal peuvent courir un risque accru d'être atteintes d'un cancer ovarien.

- Quoique les statistiques ayant trait aux conséquences du cancer de l'ovaire ne soient guère reluisantes, il faut se rappeler l'unicité de l'expérience de chaque femme. Il importe donc de faire preuve de sensibilité dans le cadre des communications avec les patientes.

\section{Approches d'évaluation}

L'évaluation de l'apprentissage des étudiants a été effectuée en administrant un pré-test et un post-test. Avant que ne débute la séance en salle de cours, on demandait aux étudiants de remplir une brève enquête et de la remettre à l'animatrice de la séance. Personne ne devait inscrire son nom sur les feuilles. Après la présentation par les survivantes et la discussion collective, on demandait aux étudiants de remplir une seconde enquête et de la remettre à l'animatrice. Celle-ci envoyait toutes les feuilles de données à la coordonatrice de la recherche à Toronto.

Les étudiants étaient également invités à remplir une seconde enquête post-test-en ligne cette fois-six mois après la tenue de la séance en salle de cours. Cette enquête post-test en ligne a été mentionnée à la fin de la séance et un rappel électronique a été envoyé aux étudiants six mois plus tard. Ceux-ci ont alors reçu une adresse Internet pour accéder à l'enquête en ligne.

Les enquêtes ont été conçues expressément aux fins de cette évaluation. Le questionnaire des enquêtes pré- et post-séance s'intéressait avant tout aux connaissances liées au cancer de l'ovaire, aux facteurs de risque, aux symptômes, aux approches d'évaluation initiale et à la compréhension des inquiétudes des patientes. 
Ces domaines représentaient 11 questions (voir les tableaux 3 et 4). Le questionnaire de l'enquête post-séance incluait également trois questions supplémentaires sur l'efficacité de la démarche pédagogique. Pour les réponses, les options étaient les suivantes : Tout à fait d'accord, D'accord, En désaccord, Tout à fait en désaccord ainsi que l'option Incertain(e). Un autre item demandait aux étudiants d'énumérer les symptômes du cancer de l'ovaire et ceux-ci avaient la possibilité d'inscrire une réponse ouverte à la fin du questionnaire. L'enquête en ligne avait exactement le même contenu que l'enquête administrée immédiatement après la séance.

\section{Analyse}

Une analyse a été effectuée pour chaque classe prise dans son ensemble. L'analyse des résultats des enquêtes pré-post séance était de nature descriptive. Les distributions statistiques et les pourcentages pour chacune des réponses aux items ont été calculés séparément pour les étudiants en médecine et ceux en sciences infirmières. La Gynecologic Cancer Foundation (2007) des É.-U. a annoncé un consensus national sur les symptômes du cancer de l'ovaire : ballonnement, douleur pelvienne ou abdominale, difficulté à manger ou sensation d'être rapidement pleine, symptômes urinaires (mictions urgentes ou fréquentes). Ces quatre symptômes ont servi à coder les données de l'item de l'enquête qui demandait aux étudiants d'« énumérer trois symptômes qui vous amèneraient à penser qu'il s'agit du cancer de l'ovaire si la femme les éprouvait de manière persistante. » Lorsque l'étudiant répondait à la question en fournissant un des quatre symptômes ci-des- sus, sa réponse était encodée " Oui ». Le contenu de la réponse à la question ouverte était passé en revue et les différentes réponses, fusionnées. Les commentaires étaient regroupés en fonction d'une analyse de contenu simple des perspectives communes.

\section{Résultats}

\section{Échantillon et taux de réponse}

Entre l'automne 2006 et mars 2009, des séances STS ont été présentées dans sept facultés de médecine et douze facultés de sciences infirmières du Canada. Trente-cinq séances ont été offertes à 798 étudiants en médecine; 35 séances ont été données à 2822 étudiants en sciences infirmières. En tout, 94 survivantes bénévoles ont été formées à la présentation des séances STS à l'échelle du Canada. À l'heure actuelle, 78 bénévoles participent activement au programme STS. L'érosion des effectifs est avant tout la conséquence directe du haut taux de mortalité chez les bénévoles.

Les taux de réponse généraux pour les enquêtes pré- et postséance se montaient à 41,4\% et 43,0\%, respectivement (voir le tableau 1). Le taux de réponse pour l'enquête de suivi en ligne aux six mois s'élevait à $19 \%$ des participants qui avaient fourni une adresse électronique lors de l'enquête initiale réalisée en salle de cours.

\section{Connaissances}

En ce qui concerne l'ensemble des items liés aux connaissances, la proportion des répondants qui donnaient des réponses justes augmentait après la séance de présentation en salle de cours. Le tableau 2 illustre la proportion de répondants qui étaient d'accord

Tableau 2. Connaissances relatives au cancer de l'ovaire : avant et après la séance

\begin{tabular}{|c|c|c|c|c|}
\hline \multirow{3}{*}{ Item } & \multicolumn{2}{|c|}{ Étudiants en médecine } & \multicolumn{2}{|c|}{$\begin{array}{l}\text { Étudiants en sciences } \\
\text { infirmières }\end{array}$} \\
\hline & \multicolumn{2}{|c|}{$\begin{array}{l}\text { Réponses Tout à fait d'accord } \\
\text { / D'accord (\%) }\end{array}$} & \multicolumn{2}{|c|}{$\begin{array}{l}\text { Réponses Tout à fait d'accord } \\
\text { / D'accord (\%) }\end{array}$} \\
\hline & Pré- (N=344) & Post- $(\mathrm{N}=352)$ & Pré- (N=1156) & Post- $(\mathrm{N}=1203)$ \\
\hline $\begin{array}{l}\text { De manière générale, j'ai une bonne connaissance de base du cancer de } \\
\text { l'ovaire. }\end{array}$ & 77,5 & 96,3 & 53,7 & 97,5 \\
\hline $\begin{array}{l}\text { Bien que le cancer de l'ovaire soit relativement rare, il constitue l'une } \\
\text { des } 5 \text { premières causes de décès par cancer chez les Canadiennes. }\end{array}$ & 87,4 & 96,0 & 73,6 & 92,6 \\
\hline $\begin{array}{l}\text { Les femmes reçoivent un test de dépistage pour le cancer de l'ovaire } \\
\text { durant un examen annuel de routine. }\end{array}$ & 8,1 & 11,1 & 26,0 & 13,0 \\
\hline $\begin{array}{l}\text { La majorité des cas de cancer ovarien sont diagnostiqués à un stade } \\
\text { précoce. }\end{array}$ & 3,8 & 2,2 & 9,8 & 5,5 \\
\hline $\begin{array}{l}\text { Dans ces stades précoces, le cancer de l'ovaire déclenche des } \\
\text { symptômes. }\end{array}$ & 7,3 & 36,1 & 8,9 & 58,8 \\
\hline $\begin{array}{l}\text { Une femme ayant des antécédents familiaux de cancer du sein, de } \\
\text { cancer du colon ou de cancer de l'ovaire court un risque accru de } \\
\text { développer un cancer de l'ovaire. }\end{array}$ & 95,6 & 98,0 & 85,0 & 97,6 \\
\hline $\begin{array}{l}\text { Une femme qui a eu le cancer du sein court un risque accru de } \\
\text { développer un cancer de l'ovaire. }\end{array}$ & 90,4 & 93,5 & 65,0 & 91,5 \\
\hline $\begin{array}{l}\text { Si je soupçonne l'une de mes patientes d'être porteuse d'un cancer de } \\
\text { l'ovaire, je la référerai à un(e) gynéco-oncologue. }\end{array}$ & 90,7 & 98,3 & 82,5 & 98,6 \\
\hline $\begin{array}{l}\text { Je peux améliorer la survie d'une femme atteinte d'un cancer de } \\
\text { l'ovaire en étant au courant de ses facteurs de risque et de ses } \\
\text { symptômes précoces. }\end{array}$ & 94,8 & 99,7 & 94,4 & 99,9 \\
\hline $\begin{array}{l}\text { Je comprends ce que peut ressentir une femme lorsqu'elle est } \\
\text { diagnostiquée d'un cancer de l'ovaire. }\end{array}$ & 52,2 & 97,9 & 47,2 & 93,5 \\
\hline
\end{tabular}


avec chaque énoncé relatif aux connaissances. Il est évident que les étudiants ont acquis une excellente compréhension et une excellente appréciation des perspectives des survivantes et des défis auxquels elles font face. Ils ont également amélioré leurs connaissances sur les principaux symptômes (tableau 3). Pourtant, il est intéressant de noter que les réponses aux items concernant l'existence d'un test de dépistage pour le cancer de l'ovaire et la présence des symptômes aux stades précoces de la maladie continuent de refléter des lacunes dans les connaissances.

\section{Efficacité de la démarche pédagogique}

Les étudiants en médecine et les étudiants en sciences infirmières indiquaient qu'ils appréciaient grandement l'efficacité de la démarche pédagogique (voir le tableau 4). Ils trouvaient que les récits des survivantes sur leurs expériences les aidaient à apprendre les symptômes et à communiquer avec les patients. Les deux groupes d'étudiants jugeaient que la présentation donnée par des survivantes complétait bien leurs études universitaires. Voici quelques-uns des commentaires écrits formulés par des étudiants :

- J'aurai conscience des symptômes précoces, ce qui fait que dans mon rôle d'infirmière, je pourrai aider mes patients à faire ce qu'il faut pour voir un médecin.

- Cela me sensibilisera aux différentes façons dont le cancer de l'ovaire peut se présenter-je réalise l'importance de faire un suivi des symptômes vagues.

- Écouter la patiente et prendre note des signes du cancer de l'ovaire-approfondir les recherches, ne pas assumer qu'il s'agit de maux d'estomac.

- Communiquer plus efficacement à l'avenir.

- Ceci m'a aidée à réaliser l'importance de la communication avec les patients et du degré d'empathie qu'il convient de manifester.

- Je n'ignorerai pas les inquiétudes de mes patients. J'accorderai toute mon attention à mes patients et j'écouterai ce qu'ils ont à dire.

- J'essaierai de me mettre dans les souliers de mes patients-je manifesterai de la sensibilité lors du diagnostic et du traitement.

- Ceci influencera la façon dont j'aborderai la communication de renseignements délicats à mes patients.

- Grâce à la présentation, je suis consciente, en tant qu'infirmière, des symptômes et de ce que les gens atteints de cancer ressentent, je pourrai donc manifester une meilleure compréhension vis-à-vis des patients.

\section{Discussion}

Le programme Survivors Teaching Students était déjà salué comme une grande réussite aux États-Unis lorsque COC a décidé de l'introduire dans le contexte canadien. L'adaptation d'un programme existant était perçue comme une solution raisonnable permettant d'épargner des efforts et d'offrir le programme plus tôt que si l'on élaborait un programme complètement neuf en partant

\begin{tabular}{|c|c|c|c|c|}
\hline \multicolumn{5}{|l|}{ Tableau 3.} \\
\hline \multirow{3}{*}{$\begin{array}{l}\text { Symptômes } \\
\text { énumérés }\end{array}$} & \multicolumn{2}{|c|}{ Étudiants en médecine } & \multicolumn{2}{|c|}{$\begin{array}{c}\text { Étudiants en sciences } \\
\text { infirmières }\end{array}$} \\
\hline & $\begin{array}{c}\text { Pré-séance } \\
(\mathrm{N}=344)\end{array}$ & $\begin{array}{c}\text { Post- } \\
\text { séance } \\
(\mathrm{N}=352)\end{array}$ & $\begin{array}{c}\text { Pré- } \\
\text { séance } \\
(\mathrm{N}=1156)\end{array}$ & $\begin{array}{c}\text { Post- } \\
\text { séance } \\
(\mathrm{N}=1203)\end{array}$ \\
\hline & \% correct & \% correct & \% correct & \% correct \\
\hline $1^{\mathrm{er}}$ symptôme & 57,3 & 60,5 & 31,9 & 58,4 \\
\hline 2e symptôme & 31,1 & 41,2 & 14,0 & 40,1 \\
\hline 3e symptôme & 18,3 & 30,1 & 7,6 & 26,7 \\
\hline
\end{tabular}

de zéro. Une évaluation formelle du programme canadien revêtait une grande importance puisqu'aucune évaluation n'avait été intégrée dans les diverses incarnations du programme américain. COC a pris l'engagement d'assurer l'efficacité de ses programmes et donc d'évaluer l'ensemble de ses programmes au fur et à mesure de leur mise en ouvre.

Le format de l'évaluation, des enquêtes pré- et post-séance de présentation, a donné des résultats assez satisfaisants pour ce qui est de la rétroaction sur le programme. Le taux de réponse était acceptable (Balajti, Daragó, Adá \& Kósa, 2010). Malheureusement, l'approche stipulant une enquête de suivi aux six mois n'a pas donné d'aussi bons résultats. La principale raison de l'échec de cette approche était que beaucoup d'étudiants n'avaient pas fourni d'adresse électronique à la fin de la séance. À ceux qui avaient laissé une telle adresse, on a envoyé un rappel pour participer à l'enquête de suivi aux six mois. Toutefois, une proportion assez importante de ces messages de rappel nous sont revenus comme étant non délivrés. Cela illustre bien la mobilité du corps étudiant. Du fait de la faible participation à l'enquête aux six mois, nous pouvons seulement discuter des résultats concernant les effets immédiats de la séance en salle de cours (enquêtes pré-/postséance).

Les présentations par des survivantes dans des salles de cours de médecine et de sciences infirmières donnaient aux étudiants une perspective particulière, et ces derniers y voyaient une démarche pédagogique efficace. Ils indiquaient qu'ils avaient désormais une vraie personne-un visage et une voix-à qui associer une histoire de cancer de l'ovaire. On pourrait faire valoir que les étudiants se souviendraient longtemps de cette expérience et qu'elle influencerait leur futur comportement en matière de pratique professionnelle. La personne qui a traversé l'épreuve du cancer confère un élément de crédibilité aux commentaires et aux récits

Tableau 4. Efficacité des stratégies d'enseignement

\begin{tabular}{|l|c|c|}
\hline \multirow{2}{*}{$\begin{array}{l}\text { Items de l'enquête } \\
\text { post-séance }\end{array}$} & Médecine (N=352) & $\begin{array}{c}\text { Sciences infirmières } \\
\text { (N=1203) }\end{array}$ \\
\cline { 2 - 3 } & $\begin{array}{c}\text { \% de réponses Tout } \\
\text { à fait d'accord / } \\
\text { D'accord }\end{array}$ & $\begin{array}{c}\text { \% de réponses Tout } \\
\text { à fait d'accord / } \\
\text { D'accord }\end{array}$ \\
\hline $\begin{array}{l}\text { Les récits des } \\
\text { survivantes } \\
\text { m'ont enseigné } \\
\text { efficacement les } \\
\text { symptômes et les } \\
\text { facteurs de risque } \\
\text { du cancer de } \\
\text { l'ovaire. }\end{array}$ & \\
\hline $\begin{array}{l}\text { Les récits des } \\
\text { survivantes } \\
\text { m'ont aidé(e) } \\
\text { à comprendre } \\
\text { l'importance de la } \\
\text { manière dont je } \\
\text { communique de } \\
\text { l'information à mes } \\
\text { patients. }\end{array}$ & 94,6 & \\
\hline $\begin{array}{l}\text { Cette présentation } \\
\text { complétait bien } \\
\text { mes études } \\
\text { universitaires sur le } \\
\text { cancer de l'ovaire. }\end{array}$ & & \\
\hline
\end{tabular}


qui sont partagés et peut être perçue comme une « championne » de la cause des personnes atteintes. L'utilisation de champions locaux est perçue comme une stratégie efficace de transfert des connaissances (Wright, Simunovic, Coates \& Fitch, 2007). À condition que la formation ait été effectuée, la présentation constitue une stimulante expérience de première main. Cependant, l'hypothèse concernant le changement des pratiques n'a pas fait l'objet d'une mise à l'épreuve particulière dans le cadre de la présente évaluation.

Les résultats de l'évaluation révèlent une amélioration des connaissances chez les étudiants. Celle-ci concerne notamment l'évolution du cancer de l'ovaire, ses symptômes, les facteurs de risque afférents et les perspectives relatives à ce que les femmes atteintes d'un cancer ovarien peuvent ressentir lorsque le diagnostic leur est communiqué. Les connaissances relatives au dépistage du cancer de l'ovaire et à la présence de symptômes au stade précoce de la maladie n'ont pas connu une aussi nette amélioration que les autres domaines de connaissances. Il est fort difficile de présenter clairement l'information visant à faire la distinction entre les concepts du dépistage dans des populations en bonne santé par rapport à la détection précoce par le biais de l'identification de symptômes. Les étudiants ont clairement indiqué que leur compréhension des perspectives des femmes avait été influencée par la présentation et les interactions.

\section{Implications}

Le recrutement et la formation des survivantes bénévoles constituent des aspects essentiels de ce programme. En particulier, la préparation des survivantes au partage de leur histoire est un élément capital de leur degré de confort et de leur aptitude à partager leur récit en toute efficacité. Sans cette formation, il est possible d'affirmer que les survivantes ne seraient pas capables de communiquer les messages clés avec la même efficacité. À l'avenir, toutefois, il faudra peut-être mettre davantage l'accent sur l'information que

\section{Références}

Andersen, M.R., Goff, B.A., Lowe, K.A., Scholler, N., Bergan, L., Dresher, C.W., ... Urban, N. (2008). Combining a symptoms index with CA 125 to improve detection of ovarian cancer. Cancer, 113(3), 484-489.

Balajti, I., Daragó, L., Adány, R., \& Kósa, K. (2010). College students' response rate to an incentivized combination of postal and webbased health survey. Evaluation and the Health Profession, 33, 164-176.

Bankhead, C.R., Collins, C., Stokes-Lampard, H., Rose, P., Wilson, S., Clements, A., ... Austoker, J. (2008). Identifying symptoms of ovarian cancer: A qualitative and quantitative study. British Journal of Obstetrics and Gynecology, 115(8), 1008-1014.

Devlin, S.M., Diehr, P.H., Andersen, M.R., Goff, B.A., Tyree, P.T., \& Lafferty, W.E. (2010). Identification of ovarian cancer symptoms in health insurance claims data. Journal of Women's Health, 19(3), 381-389.

Fitch, M.I., Gray, R.E., DePetrillo, D., Franssen, E., \& Howell, D. (1999). Canadian women's perspectives on ovarian cancer. Cancer Prevention and Control, 3(1), 52-60.

Fitch, M., Deane, K., Howell, D., \& Gray, R.E. (2002). Le vécu du cancer ovarien : réflexions sur la phase diagnostique. Revue canadienne de soins infirmiers en oncologie, 12(3), 160-168.

Goff, B.A., Mandel, L.S., Melancon, C.H., \& Muntz, H.G. ( 2004). Frequency of symptoms of ovarian cancer in women presenting to primary care clinics. Journal of the American Medical Association, 291(22), 2705-2712.

Gray, R.E., Chart, P., Carroll, J.C., Fitch, M.I., \& Cloutier-Fisher, D. (1999). Family physicians' perspectives on ovarian cancer. Cancer Prevention and Control, 3(1), 61-67. les survivantes présentent relativement au dépistage du cancer de l'ovaire et à l'apparence des signes et symptômes dès les premiers stades de la maladie, et sur la clarification de cette information.

Étant donnée la réussite du programme STS, laquelle est confirmée par les résultats de l'évaluation, on s'attend à ce que d'autres établissements de médecine et de sciences infirmières soient prêts à incorporer ce genre d'expérience d'apprentissage pour leurs étudiants. En outre, les étudiants d'autres facultés telles que celles de pharmacie, de travail social et de psychologie pourraient tirer parti d'une exposition similaire au programme. Du fait du vieillissement de la population du Canada et de la vulnérabilité accrue au cancer qui l'accompagne, les fournisseurs de soins de santé de demain se doivent d'être au courant des perspectives des survivantes et des enjeux entourant la détection précoce relativement au cancer. Sans aucun doute, le défi sera de trouver au sein des programmes d'études de premier cycle le temps exigé par l'insertion de programmes spécifiquement liés au cancer. Un autre défi de taille sera de trouver un bon équilibre entre la présentation de connaissances et d'une séance expérientielle reliées à un cancer particulier d'une part, et comment cela peut s'appliquer à l'ensemble des sièges de cancer, d'autre part.

Enfin, bien que le programme STS s'adresse aux étudiants de premier cycle, il pourrait aussi être d'une grande efficacité pour l'éducation continue des professionnels de la santé déjà en exercice. Le groupe des survivantes du cancer connaissant une croissance régulière, nous pourrions disposer d'un bassin important de candidates à la formation. Néanmoins, un tel programme devrait créer des messages clés à l'intention de ses auditoires de professionnels de la santé en fonction du point de la trajectoire du cancer où ceux-ci interagissent avec les patients. Par exemple, les infirmières en oncologie tireraient davantage profit des aspects des perspectives des survivantes concernant les professionnels de la santé que de ceux portant sur les symptômes liés à la détection précoce.

Gynecologic Cancer Foundation. (2007). Women's Cancer Network Symptoms Consensus Statement. Retrieved from http://www.wcn.org/articles/types_of_cancer/ovarian/symptoms/ concensus_statement.html

Hamilton, W., Peters, T.J., Bankhead, C., \& Sharp, D. (2009). Risk of ovarian cancer in women with symptoms in primary care: Population-based case-control study. British Medical Journal, 339, article number b2998.

Institut national du cancer du Canada [INCC] (2009). Statistiques canadiennes sur le cancer 2009 (Monographie). Toronto : Auteur.

Jayde, V., White, K., \& Blomfield, P. (2009). Symptoms and diagnostic delay in ovarian cancer: A summary of the literature. Contemporary Nurse, 34(1), 55-65.

McAndrew, A., Fitch, M., Turner, F., \& Ross, E. (2008). Cancer survivors teaching students in health care. Supportive Care in Cancer, 16, 749(\#22-241).

Rossing, M.A., Wicklund, K.G., Cushing-Haugen, K.L., \& Weiss, N.S. (2010). Predictive value of symptoms for early detection of ovarian cancer. Journal of the National Cancer Institute, 102(4), 222-229.

Ryerson, A.B., Eheman, C., Burton, J., McCall, N., Blackman, D., Subramanian, S., \& Richardson, L.C. (2007). Symptoms, diagnoses, and time to key diagnostic procedures among older U.S. women with ovarian cancer. Obstetrics \& Gynecology, 109(5), 1053-1061.

Wright, F.C., Simunovic, M., Coates, A., \& Fitch, M.I. (2007). The quality initiative in rectal cancer surgery: A qualitative study of participating surgeons. Journal of American College of Surgeons, 203(6), 795-802. 